\title{
Oriental Lacquers IX. Polymerizable Ultraviolet Stabilizers for Oriental Lacquers
}

\author{
Jan BARTus, ${ }^{*}$ William J. Simonsick, Jr., ${ }^{* *}$ and Otto VoGL* \\ * Polytechnic University, Six Metro Tech Center. \\ Brooklyn, N.Y. 11201, U.S.A. \\ ** Marshall R\&D Laboratory, E. I. Du Pont de Nemours and Co., \\ Philadelphia, PA 19146, U.S.A.
}

(Received January 9, 1995)

\begin{abstract}
This paper describes interesting results based on the preparation and utilization of UV stabilizers for Urushi. Ultraviolet stabilizers suitable for protecting coatings of oriental lacquers against the damaging effects of UV radiation were synthesized. 2-(2-Hydroxy-3-tertiarybutyl-5-[3'-hydroxypropyl]phenyl)- $2 H$-benzotriazole (Bzt) was esterified with three unsaturated carboxylic acids: oleic, linoleic, and linolenic acid. The benzotriazole derivatives were characterized by their molecular weight ( $\mathrm{K}^{+}$IDS mass spectrum), by their UV spectra and by their gas chromatographic behavior. The compounds were added to three types of oriental lacquer and the compositions were cured. The curing consists of the copolymerization of the active urushi compound, mostly trienes, with the polymerizable UV stabilizers. Simulated testing of the coatings of cured oriental lacquer containing these polymerizable UV stabilizers by exposing the coatings to intensive white light showed that the UV stabilizer containing coatings were several fold more stable than unstabilized urushi coatings.

KEY WORDS Oriental Lacquers / Ultraviolet Stabilizers / 2-(2-Hydroxyphenyl)- $2 H$-benzotriazole / Linseed Oil / $\mathrm{K}^{+}$IDS Mass Spectrometry /
\end{abstract}

For centuries oriental lacquers have been used in East Asia to coat objects of art but also objects of every day utility. ${ }^{1}$ In China oriental lacquerware has been used for several thousands of years. The coatings are prepared from the sap of the varnish tree. The varnish tree grows in the many locations in East and South-East Asia and has several subspecies. The sap of the Japanese lacquer tree (Toxicodendron vernicifluum, STOKES) is an aqueous emulsion and contains urushiol as the main component in the oily portion of the sap. The composition of urushiol may vary depending on growing conditions of the lacquer tree and on the season. The main components (about $80 \%$ ) are catechol derivatives substituted in the 3-position with unsaturated $\mathrm{C}_{15}$ linear aliphatic side chains. ${ }^{2}$ A smaller amount (perhaps $3 \%$ ) of catechol derivatives with $\mathrm{C}_{17}$ linear aliphatic side chains has also been identified. ${ }^{2}$ The side chains consist of about $70 \%$ trienes, $20 \%$ monoenes, and a smaller amount of dienes with very specific configurations of the double bonds. The presence of the trienes is essential for the effectiveness of the oxidative curing of oriental lacquers. $^{3}$

We investigated the composition of several samples of urushi ${ }^{4-6}$ and found, in addition to the usual components, one new compound. This compound is an artifact and is apparently produced by the addition of one molecule of water to the 8-cis-double bond of the predominant triene in a highly stereo- and regiospecific reaction during the processing of crude urushi. ${ }^{7}$ Freshly harvested and untreated urushi does not contain any of this compound.

The major single component of urushi as documented on "Kuro-urushi" is 3-(8-cis-11- 
trans-13-cis-pentadecatrienyl)catechol [or more correctly called $3-\left(8^{\prime} Z, 11^{\prime} E, 13^{\prime} Z\right.$-pentadecatrienyl)catechol]. The content of this compound in the urushi mixture is, according to HPLC, about 55-60\%. ${ }^{7}$ A small amount of dimers, confirmed by the mass spectroscopy, can be formed by the oxidative dimerization of the phenolic part of the urushiol components. The dimerization is known to be catalyzed by the oxidation enzyme laccase. The presence of higher oligomers was not observed in the raw nor in the processed urushi.

To make oriental lacquerware, oriental lacquers are applied on the wood from the Japanese cedar tree which is known not to warp and shrink. As the primer for the ultimate coating applications, one or two coats of unprocessed urushi are applied followed by many (as many as 30-40) thin layers of processed urushi. Each layer needs to be cured for $1-2$ days at high relative humidity (65 to $80 \%$ ) and the surface should be polished after the curing of each layer. For the final coat, a clear lacquer made from raw lacquer, is applied. The final product, a crosslinked polymer film of urushi, is resistant to water, alcohols and oils. ${ }^{8-11}$

During the curing of oriental lacquer on the wooden substrate the oxidation of the catechol portion of urushiol proceeds. Laccase, a copperglycoprotein oxido reductase enzyme, present in the sap of the lacquer tree causes the oxidative dimerization of the catechols. ${ }^{12-17}$ In addition to this reaction that couples the phenolic groups, the autooxidation of the compounds with the polyene side chains, present in the urushiol, proceeds which leads to the final lacquer film.

A similar process is involved in the curing of drying oils, such as linseed oil or tang oil, where glycerol esters of linoleic acid (octadecadienoic acid) and particularly linolenic acid (octadecatrienoic acid), are the most important components. In the oxidative curing of urushiol and of linseed oil, the methylene groups present in the 10 position of the aliphatic side chain of urushiol or the 12 position in linoleic acid, located between two double bonds, play an essential role in the effectiveness of the curing process. The hydrogen atoms of the methylene groups of these compounds are easily abstracted by free radicals. Molecular oxygen from the atmosphere is then added to the relatively stable allylic radical and the resulting hydroperoxy radical or the hydroperoxide group is the corner stone for the ensuing free radical initiated crosslinking reaction. ${ }^{18,19}$

Oriental lacquers, when applied on wood, have a beautiful appearance and an excellent stability in the normal environment. The coatings can last for centuries without noticeable aging, such as cracking, when the objects are not exposed to sunlight. Poor photooxidative stability of oriental lacquers limits their outdoor use. This can be explained by the presence of residual double bonds which, after being exposed to UV light initiate the 'post-curing' of the lacquer coating. The crosslinks that are formed during this post-curing reaction increase the crosslink density of the coating and cause shrinking of the coatings and form cracks.

We have made it our objective to develop UV stabilizers for oriental lacquers based on Bzt derivatives which can be co-cured with urushiol to oriental lacquer coatings with increased photooxidative stability. ${ }^{20}$ We chose to prepare 2-[2-hydroxy-(3'-hydroxypropyl)phenyl]- $2 H$-benzotriazole esters polymerizable groups, such as oleic, linoleic, and linolenic acids. These long unsaturated aliphatic side chains were expected to allow us oxidative co-curing of these groups with the highly unsaturated aliphatic $C_{15}$ side chains of the urushiol components.

\section{EXPERIMENTAL}

\section{Materials}

Polyethylene glycolyl 2-(2-hydroxy-3-tbutyl-5-[3'-carboxyethyl]phenyl)- $2 H$-benzotriazole [trade name Tinuvin 1130] (Ciba Geigy Corporation), lithium aluminum hydride (LAH) [95\%], (Aldrich Chemical Com- 
pany), stearic acid [95\%] (mp $\left.-67-69^{\circ} \mathrm{C}\right)$ (Aldrich Chemical Company), oleic acid [commercial] (Amend Drug \& Chemical Company), oleic acid [99\% pure] (Aldrich Chemical Company), linoleic acid [99\%] (Aldrich Chemical Company), linolenic acid [99\% pure] (Aldrich Chemical Company), $p$-toluenesulfonic acid (Eastman Organic Company), 1,1'-carbonyldiimidazole (PPG Industries), benzene (Fisher Scientific), tetrahydrofuran (Aldrich Chemical Company), aluminum oxide, activated, anhydrous, basic, Brockman I, 150 mesh (Aldrich Chemical Company), stannous octoate (New York City Chemical Corporation), sodium nitrite, (Fisher Scientific) were used as received.

Three urushi samples were obtained from the Tsutsumi Asakichi Urushi Company, Ltd.: Japanese raw urushi (Ki-urushi), Japanese transparent urushi (Suki-urushi), and Chinese black urushi (Kuro-urushi).

\section{Measurements}

$K^{+}$IDS Mass Spectrometry. For the characterization of 2-(2-hydroxyphenyl)- $2 \mathrm{H}$-benzotriazole stabilizers potassium ionization of desorped species ( $\mathrm{K}^{+}$IDS) mass spectrometry was used routinely. It allowed us to identify pure oily compounds and to analyze complicated mixtures of compounds with similar structures. The samples were measured on a Finnigan 4615B quadrupole GC/MS system using an EI source configuration. The ion source pressure (ion gauge) was less than $10^{-6}$ Torr with a source temperature of $200^{\circ} \mathrm{C}$.

UV Spectroscopy. The UV spectra of the samples were measured on a Varian Cary 2300 Spectrophotometer in a double beam mode. $n$-Hexane and chloroform were used as the solvents. The solutions were measured in a rectangular quartz cell with a path length of $10 \mathrm{~mm}$ in the range from 250 to $500 \mathrm{~nm}$. The positions of bands and corresponding absorbances were found by slowly scanning the region near the expected wavelength. The extinction coefficients $\varepsilon$ (in $1 \mathrm{~mol}^{-1} \mathrm{~cm}^{-1}$ ) were calculated according to equation

$$
\varepsilon=\frac{A}{c \cdot d}
$$

where $A$ is the absorbance at $\lambda_{\max }, c$ is the concentration of solution in mol $1^{-1}$ and $d$ is the cell thickness in $\mathrm{cm}$.

Gas chromatography was used as a qualitative method for the analysis of the purity of starting materials, reaction intermediates and of final products. A Varian 3300 Gas Chromatograph with a capillary column $(10 \mathrm{~m} \times 0.5$ mmi.d.) was used for the analysis. For higher molecular weight products (about 700 daltons), the separation of components was not complete and broad peaks were obtained even at column temperatures as high as $320^{\circ} \mathrm{C} .{ }^{6,19}$

\section{Procedures}

2-[2-Hydroxy-3-t-butyl-5(3'-hydroxypropyl)phenyl]2H-benzotriazole (Bzt). Bzt was prepared by the reduction of polyethylene glycolyl 2-(2-hydroxy-3-tertiary-butyl-5-[3'-carboxyethyl]-phenyl)- $2 H$-benzotriazole with $\mathrm{LAH}$ in anhydrous diethyl ether according of eq $1 .{ }^{21}$ The crude product was recrystallized from ether. Pure Bzt formed as white crystals and according to $\mathrm{K}^{+}$IDS mass spectrometry (Figure 1), it was almost $100 \%$ pure. The molecular peak $[\mathrm{M}] \mathrm{K}^{+}$is present at 364 daltons which corresponds to a molecular weight of 325 .

Synthesis of 2-(2-Hydroxyphenyl)2H-benzo-

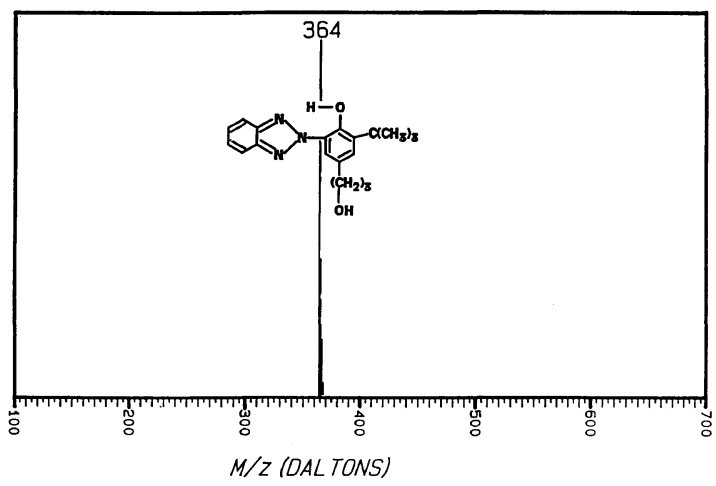

Figure 1. $\mathrm{K}^{+}$IDS mass spectrum of the 2-[2-hydroxy-3tertiary-butyl-5-(3'-hydroxypropyl)phenyl]-2H-benzotriazole. 
triazole UV Stabilizers with Long Side Chains. Bzt was allowed to react with aliphatic acids in order to obtain the corresponding esters. In the first experiments, the fatty acids, for example stearic acid was treated in anhydrous tetrahydrofuran (THF) with 1,1'-carbonyldiimidazole (CDI) to form the imidazolide. The imidazolide then was allowed to react with an alcohol (in our case the 3'-hydroxypropyl group of Bzt) to form the ester of Bzt.

For the preparation of Bzt stearate, stearic acid (2.1 g) was used together with CDI (1.5 g) in THF ( $30 \mathrm{ml})$. After 2 hours, when the carbon dioxide evolution had ceased, Bzt ( $4.8 \mathrm{~g}$ ) was added. The solution was stirred for one day at room temperature and after the evaporation of the solvent a waxy crude product was obtained. The $\mathrm{K}^{+}$IDS mass spectrum confirmed the presence of expected Bzt stearate $[\mathrm{M}] \mathrm{K}^{+}=$ 630 together with unreacted Bzt $[\mathrm{M}] \mathrm{K}^{+}=364$.

Better results were obtained when the classical esterification reaction of Bzt with the free acids was carried out in benzene using $p$ toluenesulfonic acid as the catalyst. The water formed during the reaction was removed using a Dean-Stark adapter.

In a typical procedure Bzt $(2 \mathrm{~g}, 6 \mathrm{mmol})$ was allowed to react, in a $100 \mathrm{ml} 3$-neck roundbottom flask in $15 \mathrm{ml}$ of benzene under the catalysis of $p$-toluenesulfonic acid $(0.1 \mathrm{~g})$ with a $100 \%$ excess of the aliphatic carboxylic acids (stearic oleic, linoleic, and linolenic acids). The reaction mixture was heated to reflux in an oil bath for 20 hours and the released water was collected in a Dean-Stark trap. After evaporating the benzene the crude oily products were analyzed by gas chromatography using conditions for the capillary column mentioned before. The unreacted acid was removed by column chromatography on basic aluminum oxide (column $20 \mathrm{~mm} \times 32 \mathrm{~cm}, 350 \mathrm{~g}$ of $\mathrm{Al}_{2} \mathrm{O}_{3}$ was used) with $n$-hexane as the eluent. The analysis by $\mathrm{K}^{+}$IDS mass spectrometry showed that the compounds of the purified samples were the pure Bzt esters $[\mathrm{M}] \mathrm{K}^{+}=624,626$, and 628 , respectively.

\section{RESULTS AND DISCUSSION}

We have prepared 4 esters of Bzt (eq 1) with long unsaturated aliphatic carboxylic acids. Bzt had been obtained by LAH reduction of polyethylene glycolyl 2-(2-hydroxy-3-tertiarybutyl-5-[3'-carboxyethyl]phenyl)- $2 \mathrm{H}$-benzotriazole.

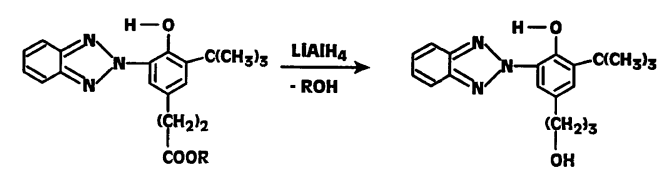

Stearic acid was used first as a model compound in order to determine the optimal conditions for the esterification reaction with Bzt. Initially, stearic acid was allowed to react with $1,1^{\prime}$-carbonyldiimidazole (CDI) in anhydrous tetrahydrofuran whereby the acid imidazolide was formed which was treated without isolation with Bzt to form the ester. The reaction product was analyzed by $\mathrm{K}^{+}$IDS mass spectrometry and was found to consist of a mixture containing a considerable amount of starting material Bzt with a $[\mathrm{M}] \mathrm{K}^{+}=364$ daltons in addition to the expected product at $[\mathrm{M}] \mathrm{K}^{+}=$ 630 daltons (Figure 2). We were not able to isolate the Bzt stearate from the crude product.

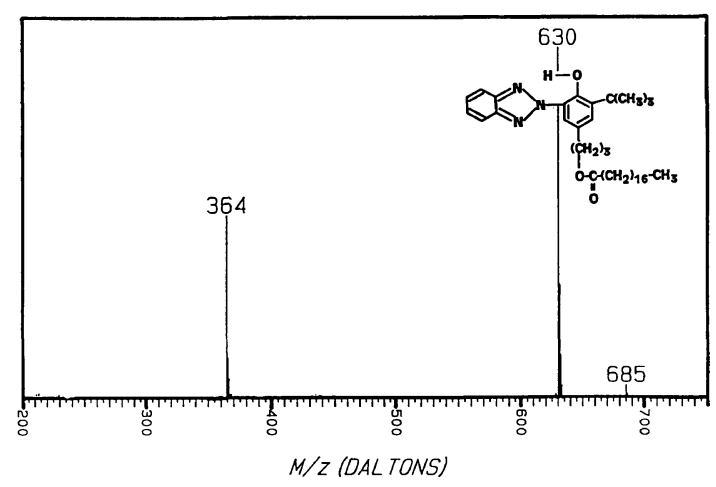

Figure 2. $\mathrm{K}^{+}$IDS mass spectrum of the 2-[2-hydroxy-3tertiary-butyl-5-(3'-hydroxypropyl)phenyl]-2H-benzotriazole reaction product with stearic acid using $1,1^{\prime}$ carbonyldiimidazole as the coupling agent. 
We next tried the classical esterification reaction for fatty acids in benzene with $p$-toluenesulfonic acid as the catalyst (eq 2) with excess of the fatty acid. Now the unreacted acid could be removed from the ester by column chromatography on basic aluminum oxide. The $\mathrm{K}^{+}$IDS mass spectrometric analysis confirmed that pure Bzt stearate $[\mathrm{M}] \mathrm{K}^{+}=630$ had been obtained. A white solid, with a $\mathrm{mp}=54-56^{\circ} \mathrm{C}$ was obtained.

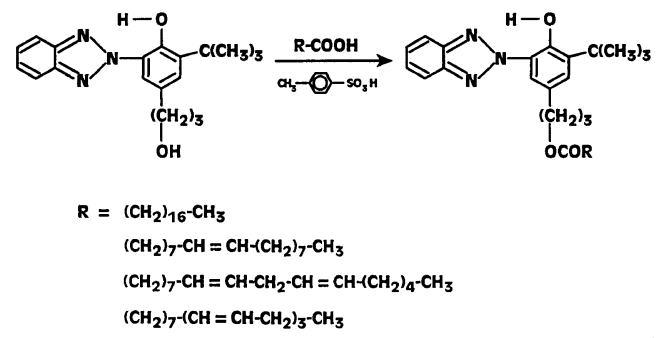

Oleic acid (initially commercial oleic acid) was next used for the esterification of Bzt. The esterification reaction gave a yellowish oily product which was subjected to purification by chromatography on an aluminum oxide column. $\mathrm{K}^{+}$IDS mass spectrometry showed the presence of a wide spectrum of components (Figure 3) with Bzt oleate as the main product at $[\mathrm{M}] \mathrm{K}^{+}=628$ daltons. In addition, we could identify the Bzt esters of palmi-

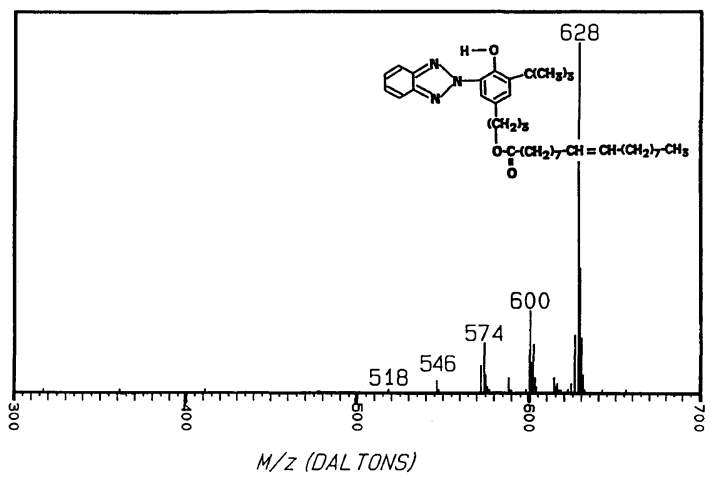

Figure 3. $\mathrm{K}^{+}$IDS mass spectrum of the 2-[2-hydroxy-3tertiary-butyl-5-(3'-hydroxypropyl)phenyl]-2H-benzotriazole reaction product with commercial oleic acid using $p$-toluenesulfonic acid as a catalyst. toleic acid at $[\mathrm{M}] \mathrm{K}^{+}=600$ daltons, of myristic acid at $[\mathrm{M}] \mathrm{K}^{+}=574$ daltons, of lauric acid at $[\mathrm{M}] \mathrm{K}^{+}=546$ daltons, and of capric acid at $[\mathrm{M}] \mathrm{K}^{+}=518$ daltons. The separation of pure Bzt oleate from the reaction mixture with the other Bzt esters was not possible.

In order to prepare pure Bzt oleate we carried out the esterification of Bzt with pure oleic acid. After the esterification reaction the excess of oleic acid was removed by a column chromatography and the $\mathrm{K}^{+}$IDS mass spectroscopic analysis confirmed that pure Bzt oleate of $[\mathrm{M}] \mathrm{K}^{+}=628$ daltons had been obtained.

Linoleic acid with one more double bond in the chain as compared to oleic acid is an important ingredient in drying oils. It is also more reactive in crosslinking reactions than the mono-unsaturated oleic acid. Esterification reaction with Bzt produced a product mixture, from which pure Bzt linoleate was obtained as a pale yellow oil (after column chromatography). The $\mathrm{K}^{+}$IDS mass spectrum confirmed the presence of Bzt linoleate at $[\mathrm{M}] \mathrm{K}^{+}=626$ daltons as the essentially sole product together with small amounts of side products (Figure 4).

Linolenic acid, octadecatrienoic acid, has a substantially increased reactivity in crosslinking reactions compared to linoleic acid and oleic acid. We had technical linolenic acid on hand but found, by $\mathrm{K}^{+}$IDS spectrometry, that

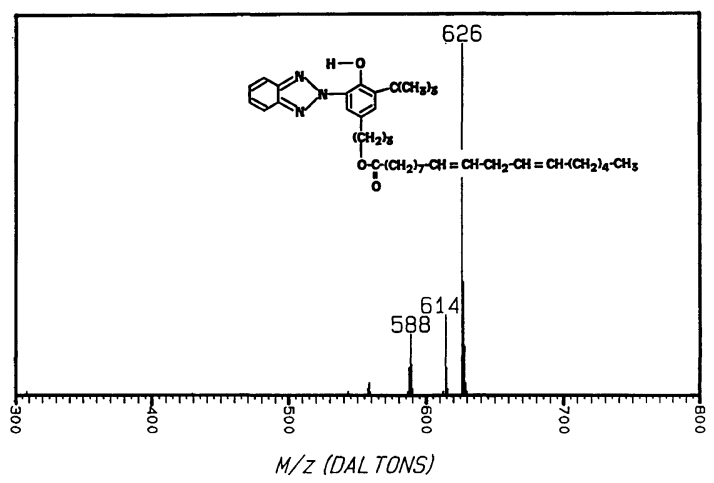

Figure 4. $\mathrm{K}^{+}$IDS mass spectrum of the 2-[2-hydroxy-3tertiary-butyl-5-(3'-hydroxypropyl)phenyl]-2H-benzotriazole reaction product with linoleic acid using $p$-toluenesulfonic acid as a catalyst. 


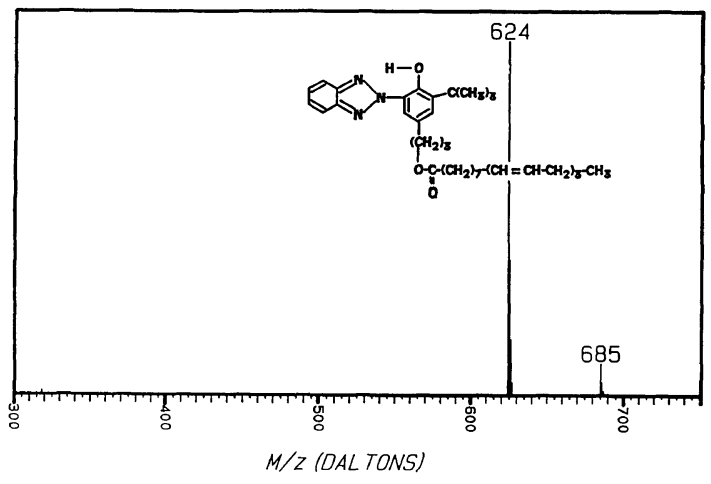

Figure 5. $\mathrm{K}^{+}$IDS mass spectrum of the 2-[2-hydroxy-3tertiary-butyl-5-(3'-hydroxypropyl)phenyl $]-2 H$-benzotriazole reaction product with linolenic acid using $p$-toluenesulfonic acid as a catalyst.

this mixture did not contain more than $10 \%$ of linolenic acid. We therefore carried out the esterification of Bzt with pure linolenic acid.

It is known that the reactivity toward oxidative crosslinking, "curing" of drying oils which are glycerol esters of oleic, linoleic, and linolenic acids increases with increasing double bond content esters with high linoleunic acid contents are particularly capable of polymerization. We were, consequently, concerned about the esterification reaction of Bzt with linolenic acid. However, after 20 hours of heating Bzt and linolenic acid in benzene the esterification was complete without formation which would have indicated polymerization of the linolenic portion of the reaction mixture. Purification of crude product on an aluminum oxide column gave, according to $\mathrm{K}^{+}$IDS mass spectrum, pure Bzt linolenate of a $[\mathrm{M}] \mathrm{K}^{+}=$ 624 (Figure 6).

Surprising was the appearance of a byproduct at $[\mathrm{M}] \mathrm{K}^{+}=685$ daltons. Its amount gradually increased as the reaction time increased. On checking earlier $\mathrm{K}^{+}$IDS spectra we found that this peak could also be found in a relatively small amount in other esterification reactions involving Bzt, including during the preparation of the Bzt stearic acid ester (Figure 2). We have thus concluded that this compound is the ester of Bzt with 2-[2-hy-

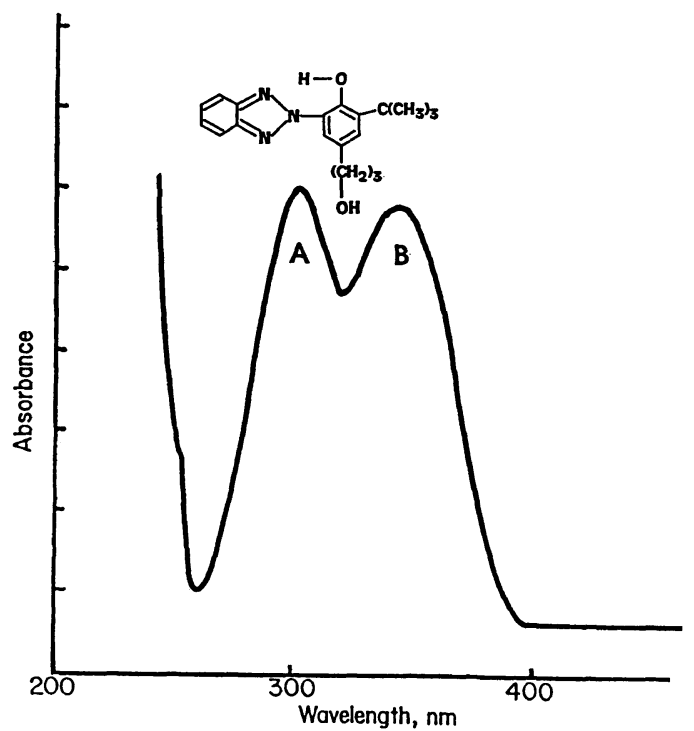

Figure 6. UV spectrum of 2-[2-hydroxy-3-tertiary-butyl5 -(3'-hydroxypropyl)phenyl]-2H-benzotriazole in chloroform.

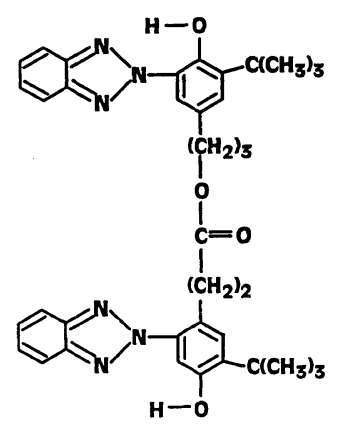

Scheme 1.

droxy-3-tertiary-butyl-5-(3'-carboxyethyl)phenyl]-2H-benzotriazole (Scheme 1).

The Bzt stabilizers prepared in this work were characterized by UV spectroscopy. It is known that the absorption band around $340 \mathrm{~nm}$ in Bzt derivatives is caused by a coplanar intramolecular hydrogen-bonded species. ${ }^{22}$ This intramolecular hydrogen bond provides a rapid nondegradative pathway for $a b-$ sorbed light energy to dissipate. ${ }^{23}$ The second band around $300 \mathrm{~nm}$ represents a non-planar species in which the intramolecular hydrogen bond is disrupted. ${ }^{22}$ This band can also be 
found in Bzt derivatives whose 2-hydroxy group is acetylated.

We measured the UV spectra of the Bzt derivatives in chloroform and $n$-hexane solution. Generally, the shape of both maxima was very similar in either of the two solvents, the only difference was the appearance of fine structures in the $240-280 \mathrm{~nm}$ region when
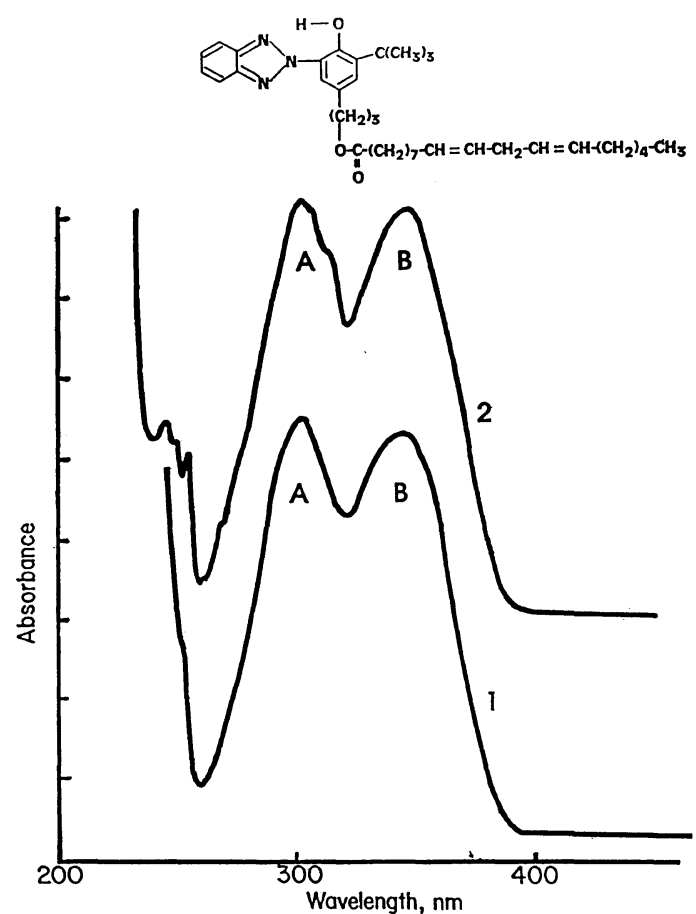

Figure 7. UV spectra of the 2-[2-hydroxy-3-tertiarybutyl-5-(3'-hydroxypropyl)phenyl]-2H-benzotriazole product with linoleic acid in chloroform (1) and in $n$-hexane (2). $n$-hexane was used as the solvent. Figure 7 shows the UV spectrum of Bzt in chloroform (Bzt is not very soluble in hexane). For comparison, the UV spectrum of Bzt linoleate in chloroform and $n$-hexane is shown in Figure 8. The values of extinction coefficients $\varepsilon$ for both maxima, calculated from UV spectra of all samples are shown in the Table I. The $\lambda_{\max }$ of the planar species $\left(\lambda_{\max } B\right)$ is slightly shifted to lower wavelengths in the Bzt esters with long aliphatic side chains. The position of the second band $\left(\lambda_{\max } \mathrm{A}\right)$ was unchanged in all cases.

We have briefly tested oriental lacquer coatings of urushi compositions that contained about $2 \%$ of Bzt stabilizers for their ability to protect the coatings of oriental lacquers against UV radiation. The "polymerizable UV stabilizers" were added to three different samples of urushi lacquers and also, for comparison, to linseed oil.

The lacquer samples, dark oily liquids, were placed in a desiccator under a nitrogen atmosphere in a refrigerator. In all cases $0.2 \mathrm{~mol} \%$ of stannous octonoate was also added, which served as a polymerization (curing) catalyst. To half of the samples $2 \mathrm{~mol} \%$ of Bzt linolenate was added, the second half served as a reference. After careful mixing for 3 min the viscous solutions were used for coatings applications.

Two base materials were used as coating substrates: polyethylene films and cedar wood. The coatings prepared on either polyethylene or wood backing were cured at room tempera-

Table I. Characterization of 2-[2-hydroxy-3-tertiary-butyl-5-(3'-hydroxypropyl)phenyl]-2H-benzotriazole (Bzt) derivatives by UV spectroscopy in chloroform

\begin{tabular}{lccccc}
\hline \multicolumn{1}{c}{ Sample type } & $\begin{array}{c}\text { Molecular } \\
\text { weight }\end{array}$ & $\begin{array}{c}\lambda_{\max } \mathrm{A} \\
\text { Wavelength/ } \\
\text { absorbance }\end{array}$ & $\varepsilon_{1}$ & $\begin{array}{c}\lambda_{\max } \mathrm{B} \\
\text { Wavelength/ } \\
\text { absorbance }\end{array}$ & $\varepsilon_{2}$ \\
\hline Bzt & 325.2 & $304 / 1.31$ & 13100 & $350 / 1.23$ & 12300 \\
Stearic acid ester & 591.7 & $304 / 1.48$ & 14800 & $339 / 1.41$ & 14100 \\
Oleic acid ester & 589.7 & $304 / 1.47$ & 14700 & $339 / 1.39$ & 13900 \\
Linoleic acid ester & 587.7 & $304 / 1.23$ & 12300 & $346 / 1.17$ & 11700 \\
Linolenic acid ester & 585.7 & $304 / 1.18$ & 11800 & $341 / 1.12$ & 11200 \\
\hline
\end{tabular}


ture and controlled humidity. It is known that the best quality of oriental lacquer curing is accomplished when the thin layers of oriental lacquer are cured at room temperature with the relative humidity of at least $65 \%$. In order to simulate these conditions we cured our films in a glass jar. The samples were placed over a Petri dish containing a saturated solution of sodium nitrite which provided the controlled humidity.

After 10 days of curing, solid shiny black coatings of the oriental lacquer were obtained on polyethylene film and on the cedar wood. From the samples prepared on the polyethylene films, small pieces $(3 \times 1 \mathrm{~cm})$ were used for UV testing. The films were fastened on the glass plate. One half of the sample was covered with an aluminum foil and one half of the sample was exposed. The glass plate was placed $10 \mathrm{~cm}$ below the 300 Watt Sylvania Sunlamp 078 (which provides a broad spectrum of white light) for 3 weeks.

After the test was judged complete the aluminum foil was removed and the quality of surface of the coatings was visually evaluated. All coatings to which the UV stabilizer had been added showed no difference in appearance between the part of the coating that had been exposed to the UV light and the part that had been covered. The lacquer coatings that did not contain the Bzt stabilizer (the originally shiny lacquer) had turned opaque on the exposed surface and visible cracks had appeared.

From our preliminary results of the "aging" studies of UV stabilized oriental lacquer compositions it is clear that the Bzt derivatives are effective UV stabilizers for oriental lacquer compositions. We have found similar results when Bzt linolenate was added to linseed oil and this composition was co-cured to UV stabilized drying oils.

\section{CONCLUSIONS}

We synthesized polymerizable UV stabilizers with linear long unsaturated aliphatic chain attached to a 2-(2-hydroxyphenyl)-2 $H$-benzotriazole moiety. The UV spectra of the synthesized 2-(2-hydroxyphenyl)- $2 \mathrm{H}$-benzotriazoles showed the presence of expected double maxima at about $340 \mathrm{~nm}$ and $300-310 \mathrm{~nm}$. The compounds were added to oriental lacquer compositions and were successfully incorporated by oxidative "co-curing" into oriental lacquer coatings to give UV-stabilized polymer films.

Our objective was to demonstrate the UV stabilization of oriental lacquer coatings by Bzt type groups. It was not our objective to evaluate in detail the effect of low molecular weight UV stabilizers $v s$. polymer-bound UV stabilizers, although this objective might in time be of considerable interest.

We believe that the demonstration that appropriately substituted Bzt type UV stabilizer could be incorporated into oriental lacquer compositions, could have a major impact on the use of urushi lacquer for outdoor uses, especially in traditional Japanese buildings, such as temples, which are the centers of great historic ad artistic importance in many locations in Japan.

Acknowledgments. This work was supported by the Herman F. Mark Chair of the Polytechnic University. We would like to thank Lutz Stoeber for the synthesis of 2-[2-hydroxy3-tertiary-butyl-5-(3'-hydroxypropyl)phenyl]- $2 H$-benzotriazole and Meifang Qin for her help during the preparation of this manuscript.

\section{REFERENCES}

1. J. Kumanotani, in "Polymer Application of Renewable-Resource Materials," C. F. Carraher and L. H. Sperling, Ed., Plenum Press, New York, N.Y., 1983, p 225 .

2. "The New Encyclopedia Britanica," Vol. 10, 15th ed, Encyclopedia Britanica Inc., Chicago, 1984, p 575.

3. M. F. Qin and O. Vogl, Cellulose Chemistry and Technology, in press.

4. O. Vogl and J. Bartus, Polym. Prepr., Am. Chem. Soc. 
Div. Polym. Chem., 33(1), 582 (1993).

5. J. Bartus, W. J. Simonsick, Jr., C. M. Garner, T. Nishiura, T. Kitayama, K. Hatada, and O. Vogl, Polym. J. (Japan), 26, 67 (1994).

6. O. Vogl, J. Bartus, M. F. Qin, and J. D. Mitchell, in "Progress in Pacific Polymer Science," Vol. 3, K. P. Ghiggino, Ed., Springer Verlag, New York, N.Y., 1994, p 423.

7. K. Hatada, K. Kitayama, T. Nishiura, A. Nishimoto, W. J. Simonsick, Jr., and O. Vogl, Macromol. Chem. Phys., 195, 1865 (1994).

8. Y. M. Du, in "Urushi," N. S. Bromelle and P. Smith, Ed., The Getty Conservation Institute, Marina del Rey, California, 1985.

9. E. Matsu, "The Chemistry of Lacquers," Daily Industry Newspaper Inc., Tokyo, 1963.

10. J. C. Y. Watt and B. B. Ford, in "East Asian Lacquer," J. P. O. Neil, Ed., The Metropolitan Museum of Art, New York, 1992.

11. B. V. Rague, "A History of Japanese Lacquer Work," University of Toronto Press, Toronto, 1976.

12. T. Kato and J. Kumanotani, J. Polym. Sci., A, 1, 1455 (1969).
13. T. Kato, Y. Yokoo, T. Taniai, and J. Kumanotani, Can. J. Chem., 47, 2106 (1969).

14. T. Kato and J. Kumanotani, Bull. Chem. Soc. Jpn., 42, 2378 (1969).

15. J. Kumanotani, Makromol. Chem., 179, 47 (1979).

16. J. Kumanotani, T. Kato, and A. Hikosaka, J. Polym. Sci., C, 23, 519 (1969).

17. R. Oshima, Y. Yamauchi, C. Watanabe, and J. Kumanotani, J. Org. Chem., 50, 2621 (1985).

18. Y. Yamauchi, R. Oshima, and J. Kumanotani, $J$. Chromatogr., 198, 49 (1980).

19. Y. Yamauchi, T. Murakami, and J. Kumanotani, $J$. Chromatogr., 214, 343 (1981).

20. O. Vogl, J. Bartus, and W. J. Simonsick, Jr., Polym. Prepr., Am. Chem. Soc. Div. Polym. Chem., 34(1), 584 (1993).

21. L. Stoeber, Ph. D. Dissertation, Polytechnic University, Brooklyn, New York, 1995.

22. R. Dux, K. P. Ghiggino, and O. Vogl, Austral. J. Chem., 47, 1461 (1994).

23. G. Woessner, G. Goeller, P. Kollat, J. J. Stezowski, M. Hauser, U. K. A. Klein, and H. E. A. Kramer, J. Phys. Chem., 88, 5544 (1984). 